\title{
ON THE PERIODIC SOLUTIONS OF THE 5-DIMENSIONAL LORENZ EQUATION MODELING COUPLED ROSBY WAVES AND GRAVITY WAVES
}

\author{
TIAGO CARVALHO ${ }^{1}$ AND JAUME LLIBRE ${ }^{2}$
}

ABSTRACT. Lorenz studied the coupled Rosby waves and gravity waves using the differential system

$$
\begin{aligned}
& \dot{U}=-V W+b V Z, \\
& \dot{V}=U W-b U Z, \\
& \dot{W}=-U V, \\
& \dot{X}=-Z, \\
& \dot{Z}=b U V+X .
\end{aligned}
$$

This system has the two first integrals

$$
\begin{aligned}
& H_{1}=U^{2}+V^{2}, \\
& H_{2}=V^{2}+W^{2}+X^{2}+Z^{2} .
\end{aligned}
$$

Our main result shows in each invariant set $\left\{H_{1}=h_{1}>0\right\} \cap\left\{H_{2}=\right.$ $\left.h_{2}>0\right\}$ there are at least 4 (respectively 2 ) periodic solutions of the differential system with $b \neq 0$ and $h_{2}>h_{1}$ (respectively $h_{2}<h_{1}$ ).

\section{INTRODUCTION}

In [3] E.N. Lorenz studied slow manifolds in coupled Rosby waves and gravity waves. In order to model the behavior he used the following 5-dimensional differential system

$$
\begin{aligned}
& \dot{U}=-V W+b V Z, \\
& \dot{V}=U W-b U Z, \\
& \dot{W}=-U V, \\
& \dot{X}=-Z, \\
& \dot{Z}=b U V+X,
\end{aligned}
$$

where $b \in \mathbb{R}$ is a parameter. In [4] it was studied the integrability of this differential system and, mainly its analytic integrability. In the present paper we shall use the first integrals of system (1) and the theory of averaging of first order for studying the periodic solutions of

2010 Mathematics Subject Classification. Primary 34C05.

Key words and phrases. Lorenz system, periodic solution, averaging theory. 
system (1), for more details on the averaging theory see $[2,5,6]$ and the appendix.

In [3] it is proved that

$$
\begin{aligned}
& H_{1}(U, V, W, X, Z)=U^{2}+V^{2}, \\
& H_{2}(U, V, W, X, Z)=V^{2}+W^{2}+X^{2}+Z^{2},
\end{aligned}
$$

are first integrals of the differential system $(1)$ when $b \neq 0$, and that

$$
\begin{aligned}
& H_{1}(U, V, W, X, Z)=U^{2}+V^{2}, \\
& H_{2}(U, V, W, X, Z)=V^{2}+W^{2}, \\
& H_{3}(U, V, W, X, Z)=X^{2}+Z^{2},
\end{aligned}
$$

are first integrals of (1) when $b=0$.

Our main results on the periodic solutions of the differential system (1) are the following.

Theorem 1. For every pair of positive real numbers $h_{1}$ and $h_{2}$ in the invariant set $\left\{H_{1}=h_{1}\right\} \cap\left\{H_{2}=h_{2}\right\}$, where $H_{1}$ and $H_{2}$ are given in (2), there are at least 4 (respectively 2) periodic solutions of the differential system (1) with $b \neq 0$ and $h_{2}>h_{1}$ (respectively $h_{2}<h_{1}$ ). We give explicit approximations of these periodic solutions and of their linear stability.

Theorem 1 is proved in section 2 .

Proposition 2. For every triple of positive real numbers $h_{1}, h_{2}$ and $h_{3}$ the general solution of differential system (1) with $b=0$ and $h_{2}-h_{1}>0$ in the invariant set $\left\{H_{1}=h_{1}\right\} \cap\left\{H_{2}=h_{2}\right\} \cap\left\{H_{3}=h_{3}\right\}$ is

$$
\begin{aligned}
& u(t)= \pm \sqrt{h_{1}} \operatorname{sn}\left(\sqrt{h_{2}-h_{1}} t \mid \frac{h_{1}}{h_{1}-h_{2}}\right), \\
& v(t)= \pm \sqrt{h_{1}-u(t)^{2}}, \\
& w(t)= \pm \sqrt{h_{2}+u(t)^{2}}, \\
& x(t)=x_{0} \cos t-z_{0} \sin t, \\
& z(t)=z_{0} \cos t+x_{0} \sin t,
\end{aligned}
$$

with $x_{0}^{2}+z_{0}^{2}=h_{3}$, and where $H_{1}, H_{2}$ and $H_{3}$ are given in (3).

In the statement of Proposition $2 s n(u \mid m)$ denotes the sinus elliptic Jacobi function whose period is $4 F(\pi / 2 \mid m)$, where $F$ is the incomplete elliptic integral of the first kind, for more details see [1].

Proposition 2 is proved in section 3, where we also provide the periodic solutions of the differential system (1) with $b=0$. 


\section{Proof of Theorem 1}

Since in this section we shall prove Theorem 1 we consider $b \neq 0$.

Analyzing the differential system (1) we obtain that the axes $U, V$ and $W$ are filled of equilibria. The Jacobian matrix $M$ of system (1) is

$$
M=\left(\begin{array}{ccccc}
0 & -W+b Z & -V & 0 & b V \\
W-b Z & 0 & U & 0 & -b U \\
-V & -U & 0 & 0 & 0 \\
0 & 0 & 0 & 0 & -1 \\
b V & b U & 0 & 1 & 0
\end{array}\right)
$$

Then the eigenvalues at the equilibrium point $(U, V, W, X, Z)=(0,0,0,0,0)$ are $0,0,0, i,-i$.

In order to simplify the next computations we shall write the linear part of system (1) at the origin of coordinates in its real Jordan normal form

$$
J=\left(\begin{array}{ccccc}
0 & -1 & 0 & 0 & 0 \\
1 & 0 & 0 & 0 & 0 \\
0 & 0 & 0 & 0 & 0 \\
0 & 0 & 0 & 0 & 0 \\
0 & 0 & 0 & 0 & 0
\end{array}\right)
$$

For this we do the linear change of variables

$$
\left(\begin{array}{l}
\bar{U} \\
\bar{V} \\
\bar{W} \\
\bar{X} \\
\bar{Z}
\end{array}\right)=\left(\begin{array}{ccccc}
0 & 0 & 0 & 1 & 0 \\
0 & 0 & 0 & 0 & 1 \\
0 & -1 & 0 & 0 & 0 \\
1 & 0 & 0 & 0 & 0 \\
0 & 0 & 1 & 0 & 0
\end{array}\right)\left(\begin{array}{c}
U \\
V \\
W \\
X \\
Z
\end{array}\right) .
$$

In the new variables $(\bar{U}, \bar{V}, \bar{W}, \bar{X}, \bar{Z})$ the differential system (1) becomes

$$
\begin{aligned}
& \dot{\bar{U}}=-\bar{V}, \\
& \dot{\bar{V}}=\bar{U}-b \overline{W X}, \\
& \dot{\bar{W}}=b \overline{V X}-\overline{X Z}, \\
& \dot{\bar{X}}=-b \overline{V W}+\overline{W Z}, \\
& \dot{\bar{Z}}=\overline{W X}
\end{aligned}
$$

whose Jacobian matrix at the origin is $J$.

Since the main tool for proving our results is the averaging theory, we need to transform the differential system (5) into the normal form (10) for applying the averaging theory, see the appendix. Thus, first we introduce in such a system a small parameter. For this we re-scale 
our variables as follows $(\bar{U}, \bar{V}, \bar{W}, \bar{X}, \bar{Z}) \rightarrow(\varepsilon u, \varepsilon v, \varepsilon w, \varepsilon x, \varepsilon z)$. Then system (5) becomes

$$
\begin{aligned}
& \dot{u}=-v, \\
& \dot{v}=u-\varepsilon b w x, \\
& \dot{w}=\varepsilon b v x-\varepsilon x z, \\
& \dot{x}=-\varepsilon b v w+\varepsilon w z, \\
& \dot{z}=\varepsilon w x .
\end{aligned}
$$

Now we shall consider the following generalized polar coordinates $(r, \theta, R, \alpha, Z)$ defined by

$$
u=r \cos \theta, v=r \sin \theta, w=R \cos \alpha, x=R \sin \alpha \text { and } z=z .
$$

Therefore the differential system (6) in these generalized polar coordinates writes

$$
\begin{aligned}
\dot{r} & =-\varepsilon \frac{1}{2} b R^{2} \sin \theta \sin (2 \alpha), \\
\dot{\theta} & =1-\varepsilon \frac{1}{2 r} b R^{2} \cos \theta \sin (2 \alpha), \\
\dot{R} & =0, \\
\dot{\alpha} & =\varepsilon(z-b r \sin \theta), \\
\dot{z} & =\varepsilon \frac{1}{2} R^{2} \sin (2 \alpha) .
\end{aligned}
$$

As consequence $R=R_{0}$, with $R_{0} \in \mathbb{R}$. As it will be clear later on, we consider $R_{0}>0$.

Now we take as new independent variable in the differential system (7) the variable $\theta$ and this system on the invariant set $\left\{H_{1}=\varepsilon^{2} R_{0}^{2}\right\}$ can be written as

$$
\begin{aligned}
r^{\prime} & =-\varepsilon \frac{1}{2} b R_{0}^{2} \sin \theta \sin (2 \alpha)+O\left(\varepsilon^{2}\right), \\
\alpha^{\prime} & =\varepsilon(z-b r \sin \theta)+O\left(\varepsilon^{2}\right), \\
z^{\prime} & =\varepsilon \frac{1}{2} R_{0}^{2} \sin (2 \alpha)+O\left(\varepsilon^{2}\right) .
\end{aligned}
$$

Here the prime denotes derivative with respect to the variable $\theta$.

First note that the differential system (8) has the additional first integral

$$
H_{2}(r, \alpha, z)=\varepsilon^{2}\left(r^{2}+z^{2}+R_{0}^{2} \cos ^{2} \alpha\right),
$$

obtained from (2) after the successive change of variables listened above. 
Now isolating $r$ from the expression of $H_{2}=\varepsilon^{2} \bar{h}_{2}>0$ we obtain that

$$
r=\sqrt{\bar{h}_{2}-z^{2}-R_{0}^{2} \cos ^{2} \alpha} .
$$

So substituting $r$ into system (8) this reduces to the system

$$
\begin{aligned}
\alpha^{\prime} & =\varepsilon\left(z-b \sqrt{\bar{h}_{2}-z^{2}-R_{0}^{2} \cos ^{2} \alpha} \sin \theta\right)+O\left(\varepsilon^{2}\right), \\
z^{\prime} & =\varepsilon \frac{1}{2} R_{0}^{2} \sin (\alpha) \cos (\alpha)+O\left(\varepsilon^{2}\right),
\end{aligned}
$$

on the invariant set $\left\{H_{1}=\varepsilon^{2} R_{0}^{2}=h_{1}\right\} \cap\left\{H_{2}=\varepsilon^{2} \bar{h}_{2}=h_{2}\right\}$.

Note that the differential system (9) is written into the normal form (10) for applying the averaging theory, see for more details the appendix. Using the notation of the appendix we have

$$
x=(\alpha, z), \quad t=\theta, \quad F_{1}(t, x)=F_{1}(\theta, \alpha, z),
$$

where

$$
F_{1}(\theta, \alpha, z)=\left(\begin{array}{c}
F_{11}(\theta, \alpha, z) \\
F_{12}(\theta, \alpha, z)
\end{array}\right)=\left(\begin{array}{c}
z-b \sqrt{\bar{h}_{2}-z^{2}-R_{0}^{2} \cos ^{2} \alpha} \sin \theta \\
\frac{1}{2} R_{0}^{2} \sin (\alpha) \cos (\alpha)
\end{array}\right) .
$$

We note that our differential system (9) satisfies all the assumptions of Theorem 3 of the appendix, so we can apply it.

Now we consider the averaging function (12) of the appendix

$$
f(\alpha, z)=\left(\begin{array}{c}
f_{1}(\alpha, z) \\
f_{2}(\alpha, z)
\end{array}\right)=\left(\begin{array}{c}
\int_{0}^{2 \pi} F_{11}(\theta, \alpha, z) d \theta \\
\int_{0}^{2 \pi} F_{12}(\theta, \alpha, z) d \theta
\end{array}\right)=\left(\begin{array}{c}
z \\
\frac{1}{2} R_{0}^{2} \sin (\alpha) \cos (\alpha)
\end{array}\right) .
$$

The zeros of the averaged function $f(\alpha, z)$ in the interval $[0,2 \pi)$ are $z_{1}=(0,0), z_{2}=(0, \pi / 2), z_{3}=(0, \pi)$ and $z_{4}=(0,3 \pi / 2)$.

The Jacobian $J f$ of the function $f$ at these zeros are

$$
J f\left(z_{1}\right)=J f\left(z_{3}\right)=-R_{0}^{2} \neq 0 \text { and } J f\left(z_{2}\right)=J f\left(z_{4}\right)=R_{0}^{2} \neq 0 .
$$

Clearly we need that $R_{0}>0$, otherwise the Jacobian is zero and the averaging theory does not provide information about the periodic solutions.

On the other hand, the eigenvalues associated to the zeros $z_{1}$ and $z_{3}$ are $-R_{0}$ and $R_{0}$, while the ones to the zeros $z_{2}$ and $z_{4}$ are $-i R_{0}$ and $i R_{0}$. Therefore according to statement (b) of Theorem 3 the two periodic solutions of system (9) associated to the zeros $z_{1}$ and $z_{3}$ are unstable, having a stable and an unstable invariant manifolds formed 
each one by two cylinders. While the two periodic solutions of system (9) associated to the zeros $z_{1}$ and $z_{3}$ are linear stable.

Now we go back through the changes of variables in order to estimate in the initial variables $(U, V, W, X, Z)$ how are the four periodic orbits that we found in the invariant set $\left\{H_{1}=\varepsilon^{2} R_{0}^{2}=h_{1}\right\} \cap\left\{H_{2}=\varepsilon^{2} \bar{h}_{2}=\right.$ $\left.h_{2}\right\}$.

According to statement (a) of Theorem 3 the periodic solution of system (9) associated to the zero $z_{1}=(\alpha, z)=(0,0)$ is of the form

$$
\alpha(\theta, \varepsilon)=O(\varepsilon), \quad z(\theta, \varepsilon)=O(\varepsilon) .
$$

This periodic solution in the differential system (8) is

$$
r(\theta, \varepsilon)=\sqrt{\bar{h}_{2}-R_{0}^{2}}+O(\varepsilon), \quad \alpha(\theta, \varepsilon)=O(\varepsilon), \quad z(\theta, \varepsilon)=O(\varepsilon),
$$

and in the differential system (7) becomes

$$
\begin{gathered}
r(t, \varepsilon)=\sqrt{\bar{h}_{2}-R_{0}^{2}}+O(\varepsilon), \quad \theta(t, \varepsilon)=t+O(\varepsilon), \quad R(t, \varepsilon)=R_{0}, \\
\alpha(t, \varepsilon)=O(\varepsilon), \quad z(t, \varepsilon)=O(\varepsilon) .
\end{gathered}
$$

This periodic solution in the differential system (6) writes

$$
\begin{gathered}
u(t, \varepsilon)=\sqrt{\bar{h}_{2}-R_{0}^{2}} \cos t+O(\varepsilon), \quad v(t, \varepsilon)=\sqrt{\bar{h}_{2}-R_{0}^{2}} \sin t+O(\varepsilon), \\
w(t, \varepsilon)=R_{0}+O(\varepsilon), \quad x(t, \varepsilon)=O(\varepsilon), \quad z(t, \varepsilon)=O(\varepsilon) .
\end{gathered}
$$

Now for the differential system (5) this last periodic solution writes

$$
\begin{gathered}
\bar{U}(t, \varepsilon)=\varepsilon \sqrt{\bar{h}_{2}-R_{0}^{2}} \cos t+O\left(\varepsilon^{2}\right), \bar{V}(t, \varepsilon)=\varepsilon \sqrt{\bar{h}_{2}-R_{0}^{2}} \sin t+O\left(\varepsilon^{2}\right), \\
\bar{W}(t, \varepsilon)=\varepsilon R_{0}+O\left(\varepsilon^{2}\right), \quad \bar{X}(t, \varepsilon)=O\left(\varepsilon^{2}\right), \quad \bar{Z}(t, \varepsilon)=O\left(\varepsilon^{2}\right) .
\end{gathered}
$$

Finally for our differential system (1) we get the periodic solution

$$
\begin{gathered}
U(t, \varepsilon)=O\left(\varepsilon^{2}\right), \quad V(t, \varepsilon)=-\varepsilon R_{0}+O\left(\varepsilon^{2}\right), W(t, \varepsilon)=O\left(\varepsilon^{2}\right), \\
X(t, \varepsilon)=\varepsilon \sqrt{\bar{h}_{2}-R_{0}^{2}} \cos t+O\left(\varepsilon^{2}\right), \quad Z(t, \varepsilon)=-\varepsilon \sqrt{\bar{h}_{2}-R_{0}^{2}} \sin t+O\left(\varepsilon^{2}\right),
\end{gathered}
$$

or equivalently, since $h_{1}=\varepsilon^{2} R_{0}^{2}$ and $h_{2}=\varepsilon^{2} \bar{h}_{2}$ we have that the periodic solution corresponding to the zero $z_{1}$ for the differential system (1) in the invariant set $\left\{H_{1}=h_{1}\right\} \cap\left\{H_{2}=h_{2}\right\}$ is

$$
\begin{gathered}
U(t, \varepsilon)=O(\varepsilon), \quad V(t, \varepsilon)=-\sqrt{h_{1}}+O(\varepsilon), W(t, \varepsilon)=O(\varepsilon), \\
X(t, \varepsilon)=\sqrt{h_{2}-h_{1}} \cos t+O(\varepsilon), \quad Z(t, \varepsilon)=-\sqrt{h_{2}-h_{1}} \sin t+O(\varepsilon),
\end{gathered}
$$

once we have substitute $R_{0}$ and $\bar{h}_{2}$ by $\sqrt{h_{1}} / \varepsilon$ and $h_{2} / \varepsilon^{2}$, respectively. Note that this last solution satisfies that it is on the invariant set $\left\{H_{1}=\right.$ $\left.h_{1}\right\} \cap\left\{H_{2}=h_{2}\right\}$. 
In a similar way we can study the periodic solutions of the differential system (1) corresponding to the zeros $z_{k}$ for $k=2,3,4$, and we obtain

$$
\begin{aligned}
& U(t, \varepsilon)=-\sqrt{h_{1}}+O(\varepsilon), \quad V(t, \varepsilon)=O(\varepsilon), \quad W(t, \varepsilon)=O(\varepsilon), \\
& X(t, \varepsilon)=\sqrt{h_{2}} \cos t+O(\varepsilon), \quad Z(t, \varepsilon)=-\sqrt{h_{2}} \sin t+O(\varepsilon),
\end{aligned}
$$

for the zero $z_{2}$,

$$
\begin{gathered}
U(t, \varepsilon)=O(\varepsilon), \quad V(t, \varepsilon)=\sqrt{h_{1}}+O(\varepsilon), W(t, \varepsilon)=O(\varepsilon), \\
X(t, \varepsilon)=\sqrt{h_{2}-h_{1}} \cos t+O(\varepsilon), \quad Z(t, \varepsilon)=-\sqrt{h_{2}-h_{1}} \sin t+O(\varepsilon),
\end{gathered}
$$

for the zero $z_{3}$, and

$$
\begin{gathered}
U(t, \varepsilon)=\sqrt{h_{1}}+O(\varepsilon), \quad V(t, \varepsilon)=O(\varepsilon), \quad W(t, \varepsilon)=O(\varepsilon), \\
X(t, \varepsilon)=\sqrt{h_{2}} \cos t+O(\varepsilon), \quad Z(t, \varepsilon)=-\sqrt{h_{2}} \sin t+O(\varepsilon),
\end{gathered}
$$

for the zero $z_{4}$. This completes the proof of Theorem 1 .

\section{Proof of Proposition 2}

Using an algebraic manipulator as mathematica the general solution (4) of the differential system (1) with $b=0$ is easily obtained.

We note that system (1) with $b=0$ has the axes $u, v$ and $w$ filled of equilibria.

Moreover we remark that the 3-dimensional subspace $\{x=0\} \cap\{z=$ $0\}$ and the 2-dimensional subspace $\{u=0\} \cap\{v=0\} \cap\{w=0\}$ are filled of periodic solutions if we remove the equilibria which are in these subspaces.

When the quotient $h_{1} /\left(h_{1}-h_{2}\right)$ satisfies $4 F\left(\pi / 2 \mid h_{1} /\left(h_{1}-h_{2}\right)\right)=2 \pi$, the 3-dimensional invariant sets $\left\{H_{1}=h_{1}\right\} \cap\left\{H_{2}=h_{2}\right\}$ are filled of period solutions of period $2 \pi$.

\section{ApPendix: The AVERAging TheOry of First ORDER}

Consider the differential system

$$
\dot{x}=\varepsilon F_{1}(t, x)+\varepsilon^{2} F_{2}(t, x, \varepsilon), \quad x(0)=x_{0}
$$

with $x \in D$, where $D$ is an open subset of $\mathbb{R}^{n}, t \geq 0$. We also assume that the functions $F_{1}(t, x)$ and $F_{2}(t, x, \varepsilon)$ are $T$-periodic in $t$. We define in $D$ the averaged differential system

$$
\dot{y}=\varepsilon f(y), \quad y(0)=x_{0},
$$

where

$$
f(y)=\frac{1}{T} \int_{0}^{T} F_{1}(t, y) d t
$$


The next result shows that under the convenient hypotheses the equilibria of the averaged system will provide $T$-periodic solutions of system (10).

Theorem 3. Consider the two initial value problems (10) and (11). Suppose that

(i) the functions $F_{1}, \partial F_{1} / \partial x, \partial^{2} F_{1} / \partial x^{2}, F_{2}$ and $\partial F_{2} / \partial x$ are defined, continuous and bounded by a constant independent of $\varepsilon$ in $[0, \infty) \times D$ and $\varepsilon \in\left(0, \varepsilon_{0}\right]$;

(ii) the functions $F_{1}$ and $F_{2}$ are $T$-periodic in $t$ ( $T$ independent of $\varepsilon)$.

Then the following statements hold.

(a) If $p$ is an equilibrium point of the averaged system (11) satisfying

$$
\left.\operatorname{det}\left(\frac{\partial f}{\partial y}\right)\right|_{y=p} \neq 0,
$$

then there is a $T$-periodic solution $x(t, \varepsilon)$ of system (10) such that $(0, \varepsilon) \rightarrow p$ as $\varepsilon \rightarrow 0$.

(b) The kind of stability or instability of the periodic solution $x(t, \varepsilon)$ coincides with the kind of stability or instability of the equilibrium point $p$ of the averaged system (11). The equilibrium point $p$ has the kind of stability behavior of the Poincaré map associated to the periodic solution $x(t, \varepsilon)$.

For a proof of Theorem 3, see sections $6.3,11.8$ of Verhulst [6].

\section{ACKNOWLEDGMENTS}

The first author is partially supported by a FAPESP/Brazil grant number 2014/02134-7. The second author is partially supported by a MINECO/FEDER grant MTM2008-03437 and MTM2013-40998-P, an AGAUR grant number 2014SGR568, an ICREA Academia, the grants FP7-PEOPLE-2012-IRSES 318999 and 316338, FEDER-UNAB-10-4E378 , and a CAPES grant number 88881.030454/2013-01 from the program CSF-PVE.

\section{REFERENCES}

[1] M. Abramowitz And I.A. Stegun, Handbook of mathematical functions with formulas, graphs, and mathematical tables, National Bureau of Standards Applied Mathematics Series 55, U.S. Government Printing Office, Washington, D.C. 1964.

[2] A. Buica AND J. LlibRe, Averaging methods for finding periodic orbits via Brouwer degree, Bull. Sci. Math. 128 (2004), 7-22. 
[3] E.N. Lorenz, On the existence of a slow manifold, J. Atmospheric Sci. 43 (1986), 1547-1557.

[4] J. Llibre, R. SAghin And X. Zhang, On the analytic integrability of the 5-dimensional Lorenz system for the gravity-wave activity, Proc. Amer. Math. Soc. $142(2014), 531-537$.

[5] J.A. Sanders, F. Verhulst and J. Murdock, Averaging methods in nonlinear dynamical systems, Second edition, Applied Mathematical Sciences 59, Springer, New York, 2007.

[6] F. Verhulst, Nonlinear Differential Equations and Dynamical Systems, Universitext Springer Verlag, 1996.

${ }^{1}$ Departamento de Matemática, Faculdade de Ciências, UnesP, Av. Eng. Luiz Edmundo Carrijo Coube 14-01, CEP 17033-360, Bauru, SP, BRAZIL.

E-mail address: tcarvalho@fc.unesp.br

2 Departament de Matemàtiques, Universitat Autònoma de Barcelona, 08193 Bellaterra, Barcelona, Catalonia, Spain.

E-mail address: jllibre@mat.uab.cat 